\title{
Bericht über die Februar-Sitzung 1908.
}

Verhandelt Berlin, Montag, den 3. Febr. abends 8 Uhr im Architekten-Vereinshause, Wilhelmstr. 92.

Anwesend die Herren v. Lucanus, Sch nöckel, K. Neunzig, K. Kothe, Rörig, Ehmcke, v. Treskow, Krause, Reichenow, Deditius, Heinroth.

Als Gäste die Herren v. Versen, P. Kothe, Christensen, Schou, Miethke, Le Coq und Frau Heinroth.

Vorsitzender Herr R eich en ow, Schriftfuhrer HerrH e in rot h.

Der Vorsitzende begrï Ist als neu aufgenommene Mitglieder die Herren Berger, Schnöckel und C. Kothe in Berlin, D o meier-Münden, Grote-Eberswalde, Teichmüller-Dessau, T is chler-Losgehnen (Ostpr.) und berichtet über die KuratoriumsSitzung vom 25. 1. 08 der Vogelwarte Rossitten. Er teilt mit, dafs der Leiter der Warte nunmehr staatlich angestellt sei, während das Institut selbst wie bisher unter der Verwaltung der Gesellschaft bis auf weiteres verbleibe.

Herr Reichenow bespricht die eingegangene Literatur. Herr Le Coq legt eine Anzahl von ihm in Turkestan angefertigter Photographieen vor, welche Steinadler, Habicht, Wander- u. Baumfalk als Baizvögel darstellen. Herr Heinroth gibt einige Erläuterungen zu einem kürzlich erschienenen Aufsatze über ,Trächtigkeits- und Brutdauern".

Herr Ehmcke legt hierauf 3 verschiedene Grauammern vor, die sämtlich bei Rehfelde in der Mark erlegt sind, und zwar ist die eine Form der Brutvogel, die zweite der Wintervogel, die dritte ganz abweichende und sehr kleine Form wird nur selten und zwar auch nur im Herbst und Winter angetroffen, hat auch eine andere Lebensweise als die gewöbnlichen Grauammern. Herr Ehmcke weist darauf hin, dafs die typische Emberiza calandra L. nicht der Brut-, sondern der Wintervogel in.Rehfelde sei. Über den interessanten kleinen Ammer entspinnt sich eine längere Debatte.

Herr Heinroth demonstriert hierauf die „Sammtleiste" auf der Oberseite der Innenfahne einer Caprimulgus-Hindschwinge, ein Gebilde, welches den geräuschlosen Flug dieses Vogels entstehen lälst und bespricht die interessanten ornithologischen Veränderungen und Vorgänge im Berliner Zoologischen Garten während des Jahres 1907. An Neu-Einführungen sind zu nennen: Philetaerus socius, Cicinnurus regius, Bubo vosseleri, Houbara undulata, Nettium torquatum, Rhodonessa caryophyllacea, Philacte canagica, Phalacrocorax pygmaeus und ein Paar Mischlinge von Mareca penelope mas. X Lampronessa sponsa fem. Ferner kam ein Didunculus strigirostris nach längerer Zeit wieder in den Garten. Ausgebrütet wurden unter anderem mehrere Eier eines hier befindlichen Paares Lagopus lagopus, trotz aller Mühe gelang es aber nicht, die Jungen länger als sieben Tage am Leben zu er- 
halten, ein Paar Crax carunculata erbrütete selbst ein Ei und zog das Junge vortrefflich auf. Auch im Vorjahre wurden wieder von den freifliegenden Brautenten zahlreiche Gelege in den an den Bäumen aufgehängten Nisthöhlen erzeugt und der Bestand stark vermebrt. Schliefslich beschrieb der Vortragende noch den von den Amerikanern so gerühmten Gesang des Myiadestes townsendi. Der "Klarino" macht seinem Namen alle Ehre, er singt tatsächlich mit der Klangfarbe einer Klarinette, jedoch sehr monoton und wird auf die Dauer recht langweilig.

Dr. O. Heinroth.

\section{Dem Herausgeber zugesandte Schriften.}

Annuaire du Musée Zoologique de l'Académie Impériale des Sciences de St. Pétersbourg. 1907 No. 2-3.

The Auk. A Quarterly Journal of Ornithology. Vol. XXV. No. 1. 1908.

Bulletin of the British Ornithologists' Club. No. CXXXVIIICXL. 1908.

The Ibis. A Quarterly Journal of Ornithology. (9). II. 1908. Heft 1.

Journal of the South African Ornithologists' Union. Vol. III. No. 2. 1907.

Ornithologische Monatsschrift. XXXIII. No. 1-3. 1908.

J. A. Alle n, Pennant's "Indian Zoology". (Abdruck aus: Bull. Amer. Mus. Nat. Hist. 24. Art. 5. 1908).

- A List of the Genera and Subgenera of North American Birds, with their Types, According to Article 30 of the International Code of Zoological Nomenclature. (Abdruck aus: Bull. Amer. Mus. Nat. Hist. 24. Art. 1. 1907).

W. R. Butterfield, On Subspecies in Ornithology and their Nomenclature. (Abdruck aus: Aquila 14. 1907).

F. Cerva, Nidologische und oologische Beobachtungen. (Abdruck aus: Aquila 14. 1907).

St. Chernel v. Chernelháza, Daten zur Vogelfauna Ungarns. (Abdruck aus: Aquila 14. 1907).

- Daten zur Vogelfauna Ungarns. (Abdruck aus: Aquila 14. 1907).

G. Clodius, Ornithologischer Bericht über Mecklenburg (und Lübeck) für das Jahr 1906. (Abdruck aus: Archiv Ver. Fr. Naturgesch. Meckl. 61. 1907).

Cowans's Nature Books. No. 10 Photographs by the Scottish Antarctic Expedidion. No. 13 Birds at the Zoo. No. 19 Wild Birds at Home. (Verlag: Wilh. Weicher, Leipzig Inselstr. 10). 